\title{
What Exactly is Meant by "Loss of Domain" for Ventral Hernia? Systematic Review of Definitions
}

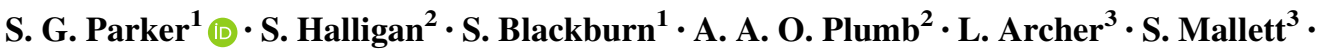 \\ A. C. J. Windsor ${ }^{1}$
}

Published online: 5 September 2018

(C) The Author(s) 2018

\begin{abstract}
Large ventral hernias are a significant surgical challenge. "Loss of domain" (LOD) expresses the relationship between hernia and abdominal volume, and is used to predict operative difficulty and success. This systematic review assessed whether different definitions of LOD are used in the literature. The PubMed database was searched for articles reporting large hernia repairs that explicitly described LOD. Two reviewers screened citations and extracted data from selected articles, focusing on the definitions used for LOD, study demographics, study design, and reporting surgical specialty. One hundred and seven articles were identified, 93 full-texts examined, and 77 were included in the systematic review. Sixty-seven articles were from the primary literature, and 10 articles were from the secondary literature. Twenty-eight articles (36\%) gave a written definition for loss of domain. These varied and divided into six broad groupings; four described the loss of the right of domain, six described abdominal strap muscle contraction, five described the "second abdomen", five describing large irreducible hernias. Six gave miscellaneous definitions. Two articles gave multiple definitions. Twenty articles $(26 \%)$ gave volumetric definitions; eight used the Tanaka method [hernia sac volume (HSV)/abdominal cavity volume] and five used the Sabbagh method [(HSV)/total peritoneal volume]. The definitions used for loss of domain were not dependent on the reporting specialty. Our systematic review revealed that multiple definitions of loss of domain are being used. These vary and are not interchangeable. Expert consensus on this matter is necessary to standardise this important concept for hernia surgeons.
\end{abstract}

Electronic supplementary material The online version of this article (https://doi.org/10.1007/s00268-018-4783-7) contains supplementary material, which is available to authorized users.

S. G. Parker

samgparker@nhs.net

1 The Abdominal Wall Unit, University College London Hospital, University College London Hospitals NHS Foundation Trust, 235 Euston Road, London NW1 2BU, UK

2 UCL Centre for Medical Imaging, Charles Bell House, 43-45 Foley Street, Fitzrovia, London W1W 7TS, UK

3 Institute of Applied Health Sciences, College of Medical and Dental Sciences, University of Birmingham, Edgbaston, Birmingham B15 2TT, UK

\section{Introduction}

The incidence of ventral hernia disease is increasing [1,2]. This is due to an ageing population [3], the obesity epidemic [4], and an increasing number of abdominal operations being performed [5]. The proportion of complex ventral hernia $(\mathrm{CVH})$ has also increased, partly due to the reasons already mentioned but also because of improvements in intensive care medicine [6]. In many patients' following intra-abdominal sepsis and laparostomy, the ventral defect is left open and covered only via skin grafting, culminating in large ventral hernia. These $\mathrm{CVH}$ contain a significant proportion of the abdominal viscera outside the abdominopelvic compartment and their repair presents the sternest surgical challenge. "Loss of domain" 
(LOD) is a term used commonly in the hernia literature to describe the distribution of abdominal content between the hernia and residual abdominopelvic cavity. After repairing hernias with significant LOD (i.e. large hernias with much of the abdominal viscera outside the abdominal compartment), serious physiological complications can arise. The increase in intra-abdominal pressure pushes up on the diaphragm and can cause respiratory failure and pneumonia. The rise in abdominal pressure increases the tension along the laparotomy incision, which can be pulled apart resulting in wound complications [7] and hernia recurrence [7]. Post-operative recurrence is a significant problem and has precipitated interest in discerning pre-operative factors that help to predict success or failure $[8,9]$. LOD may have prognostic value, and accordingly a standardised definition is warranted. A standardised definition will allow for comparable pre-operative assessment of hernia patients. Trials in hernia repair will then be able to use this definition, and subsequent trial comparison via meta-analysis will allow researchers to investigate LOD as a predictor.

Whilst reviewing the literature and holding discussions with hernia surgeons, we suspected that LOD was not being utilised in any standardised fashion. Supporting this observation, articles have suggested that written definitions of LOD are inconsistent [10]. Cross-sectional imaging with volumetric analysis is used increasingly to quantify LOD and researchers have noted that volumetric definitions of LOD can differ $[11,12]$. If LOD is to be a useful concept, then it is clear that its definition should be standardised and applied consistently. In order to progress this, we used systematic review to identify the frequency with which different definitions of LOD were presented in the literature, and examined what these were.

\section{Methods}

\section{Objectives}

The primary aim of this systematic review was to investigate the range of written and volumetric definitions for LOD used in the literature and to report the frequency of each definition used.

\section{Reporting and registration}

This systematic review was performed and reported in line with the Preferred Reporting Items for Systematic Reviews and Meta-Analyses (PRISMA) statement [13]. Ethical permission is not required by our University for systematic reviews of available primary literature.

\section{Inclusion criteria for studies}

We aimed to identify indexed studies that used the term "loss of domain" in their methods when describing the morphology of hernia. No date limitation was used for our search. There was no limit to the manuscript type, allowing for the inclusion of both the primary and secondary literature in our review. Only articles written in English were included.

\section{Target condition}

The target condition was hernia with LOD. The term LOD is frequently caused to describe large ventral hernias; however, deliberately, our search strategy did not exclude any specific subtypes or aetiologies of hernia (e.g. large inguinal or diaphragmatic hernias) as our aim was to investigate all definitions of LOD, which can be applied to hernia irrespective of hernia aetiology. We wished to encompass definitions used not only by specialist abdominal wall surgeons but also those used by general, trauma, plastic, transplant, bariatric, and paediatric surgeons.

\section{Participants}

Participants were defined as those with large hernia with LOD, either as part of a primary study or as part of a secondary narrative review or editorial. We included paediatric patients, as the literature commonly describes the surgical repair of gastroschisis and omphalocele using the term loss of domain.

\section{Search strategy and string}

The primary researcher, SGP, searched the PubMed database with no date limitation. Filters were applied limiting the search to "human studies". Our search string used the keywords; "loss of domain", "loss of abdominal domain", and "hernia". These terms were combined as two criteria to identify relevant articles:

(1) "Loss of domain" OR "Loss of abdominal domain" AND

(2) "hernia"

MESH terms were not used as "loss of domain" is indexed under multiple terms. After entering the above keywords, our search strategy was transformed to search for articles indexed under any mesh term containing the keyword "hernia" combining this with the keywords "loss" and "domain" (our search string is shown in "Appendix 1"). 


\section{Citation management and screening}

Identified citations were entered into a spreadsheet (Microsoft Excel for Mac 2011 v.14.5.9, Microsoft Corporation, Washington) and uploaded subsequently into a reference manager able to access the online original articles directly (Mendeley Desktop v 1.17.13, London, UK). After the search filters were applied and duplicates excluded, the citation titles were screened by two researchers (SGP, SB). Citations were excluded that were clearly unsuitable for full-text assessment. Where there was uncertainty between the two researchers for citation inclusion, differences were discussed by face-to-face discussion. The full-text of the remaining articles was assessed for eligibility, and articles were excluded if they were not written in English, not describing abdominal loss of domain, and if they were unavailable (even after using our institution's inter-library loan service).

\section{Data extraction}

Two researchers, SGP and SB, reviewed the full text of each article selected independently. Any data discrepancies were discussed face-to face, and if persistent they were discussed with a senior researcher. Data were extracted into an excel spreadsheet. Data extracted related to study type, year and country of publication and surgical specialty (our classification for abdominal wall specialist surgeons is shown in "Appendix 2"). Our primary aim was to extract definitions for LOD used in the literature. Our anecdotal experience was that authors used the phrase "loss of domain" as a concept to describe large hernias but without precise definition. However, any reported written and/or volumetric definitions were extracted. Free text space was also available to record any additional features regarding an individual study's definition of LOD. Where documented, we also collected authors' opinions of the "cutoff" threshold or percentage proportion above which they believed LOD became clinically significant, i.e. the point at which closing the abdomen becomes very challenging and physiological complication increasingly likely.

We deemed that studies originating from the same research group were acceptable as groups may use a different definition of LOD as the literature evolves. This also applied to studies who reported overlapping patient groups since our review concentrates on definitions rather than treatment effects.

\section{Risk of bias}

We did not assess risk of bias because we were interested in definitions of loss of domain rather than methodological quality.
Results

Our initial search retrieved 107 results (Fig. 1). After applying search filters and removing duplicates, we excluded a further 5 non-human studies, leaving 102 records for title and abstract review. After title screening, we excluded a further 9 genetics studies, leaving 93 articles for full-text review. A further 16 studies were excluded during this final stage, 7 articles couldn't be found despite attempts to obtain them using our University's inter-library loan service, 5 articles didn't describe LOD, and 4 articles were not written in English; leaving 77 articles for inclusion in the systematic review (Online supplementary resource 1 ).

The majority of the articles, 39, originated from the USA; five were from France [11, 14-17], four from the UK [12, 18-20], and two from Italy [21, 22], India [23, 24], and Brazil [25, 26]. Six manuscripts were published prior to 2000 [27-32], 20 were published between 2000 and 2009, and 51 were published from 2010 onwards. Sixty-five articles described LOD in the context of ventral hernia patients, 9 articles described LOD caused by giant inguinal hernia, and 3 articles described giant diaphragmatic hernia. Sixty-seven articles were from the primary literature, comprising 44 case series, 17 case reports, 4 retrospective database analyses [18, 33-35], and 2 retrospective interventional studies [36] [30]. Ten articles were from the secondary literature comprising seven editorials, two systematic reviews [20,37] and one consensus questionnaire [11]. The primary literature reported a total of 1528 patients; 419 of these were retrospective database analyses.

Thirty-eight of the articles were written by abdominal wall specialists, 16 articles were written by general surgeons, seven by paediatric surgeons, six by trauma surgeons, six by plastic surgeons, and two, one, and one by Transplant [38, 39], vascular [40] and bariatric [41] surgeons, respectively. Twenty-eight [36\%] of articles presented a written definition for LOD (Online supplementary resource 2), meaning that the remaining 49 [64\%] articles used the phase "loss of domain" as a concept without definition. The written definitions reported were inconsistent. Definitions varied but could be categorised into six groups (Table 1). Four out of these six groups used definitions based around four theoretical concepts. Four articles defined LOD by describing a hernia as so large that "the herniated organs have lost their right of domain inside the abdominal cavity" [15, 16, 29, 35]. Six articles use the principle of lateral contraction of the abdominal wall muscles leading to a reduced volume of the abdominal cavity and progressive visceral protrusion [39, 42-46]. Five articles use the concept of the hernia sac being a "second abdomen" and included the argument that restoring the 


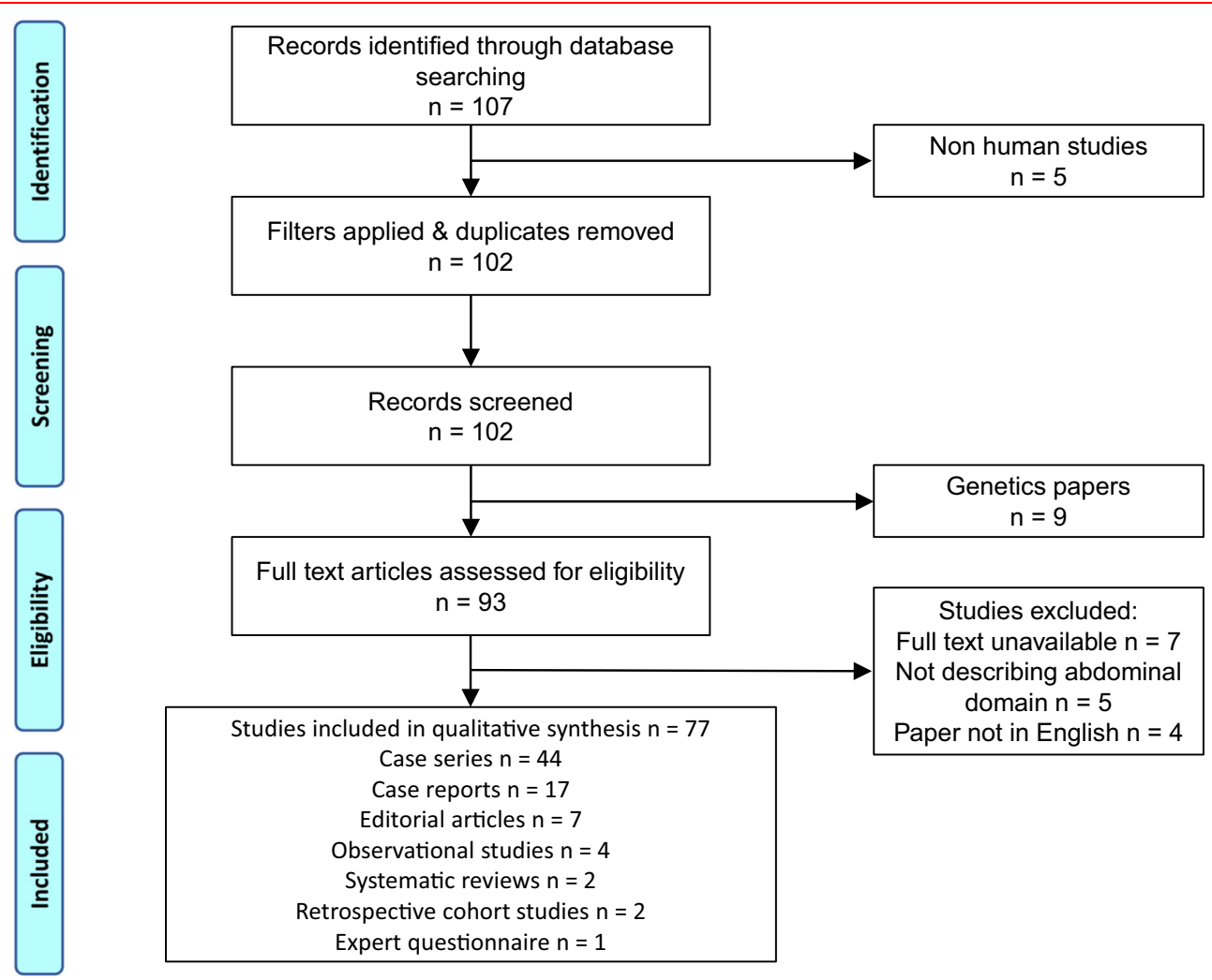

Fig. 1 PRISMA flow chart of study selection

hernia sac back into the abdominal cavity would create physiological disturbances and complications [14, 19, 25, 47, 48]. Lastly, five articles describe LOD as a large irreducible hernia containing abdominal viscera residing outside the abdominal cavity and adherent to the hernial sac [11, 26, 49-51]. Six of the definitions were miscellaneous [52-57], and two of the articles were editorials $[10,12]$, which highlighted inconsistencies when defining LOD. Twenty-three of the 28 [82\%] definitions were reported in articles written by abdominal wall specialists. After categorising results by reporting specialty, the definitions remained inconsistent and were not dependent on the reporting surgical specialty (Table 1).

Volumetric definitions used for LOD were also inconsistent. In total, 20 studies used cross-sectional imaging combined with volumetric analysis pre-operatively (Table 2). Eight studies [11, 25, 26, 44, 46, 51, 58, 59] reported the ratio of the hernia sac volume (HSV) to the abdominal cavity volume (ACV), commonly referred to as the Tanaka method [25]. Five studies [14-16, 49, 50] reported the ratio or percentage of the HSV to the total peritoneal volume $(\mathrm{TPV}=\mathrm{HSV}+\mathrm{ACV})$, known as the Sabbagh method [15]. Four of the papers describe volumetric analyses but were unclear how LOD was calculated [19, 48, 56, 60]. Finally, 2 studies calculated HSV and
ACV but simply stated these two volumes without using a ratio or a proportion $[35,61]$. One editorial review discussed both methods used to calculate LOD [12]. Only two studies $[59,61]$ using volumetric analysis were not reported by abdominal wall specialists. Therefore, a volumetric definition for LOD remained inconsistent even amongst hernia specialists (Table 2). Fifteen papers also reported a threshold at which they believed LOD became clinically significant, but this appeared anecdotal in all, based on clinical expertise rather than any independent research. Values ranged from $10 \%$ [60] to 50\% [49], with the most frequently reported value being $20 \%[15,51,58,59]$.

\section{Discussion}

Prior to discussing the findings of this review, it is necessary to recap the complex processes that underpin large ventral hernia disease. After the linea, alba is divided by a midline hernia forms, and over time the abdominal muscles retract laterally (due to mechanical unloading), and the hernia gradually enlarges. Due to disuse atrophy, irreversible muscular fibrosis follows, the muscles becoming stiffer and less elastic [10]. Consequently, the abdominal strap muscles become shorter and thicker. These 


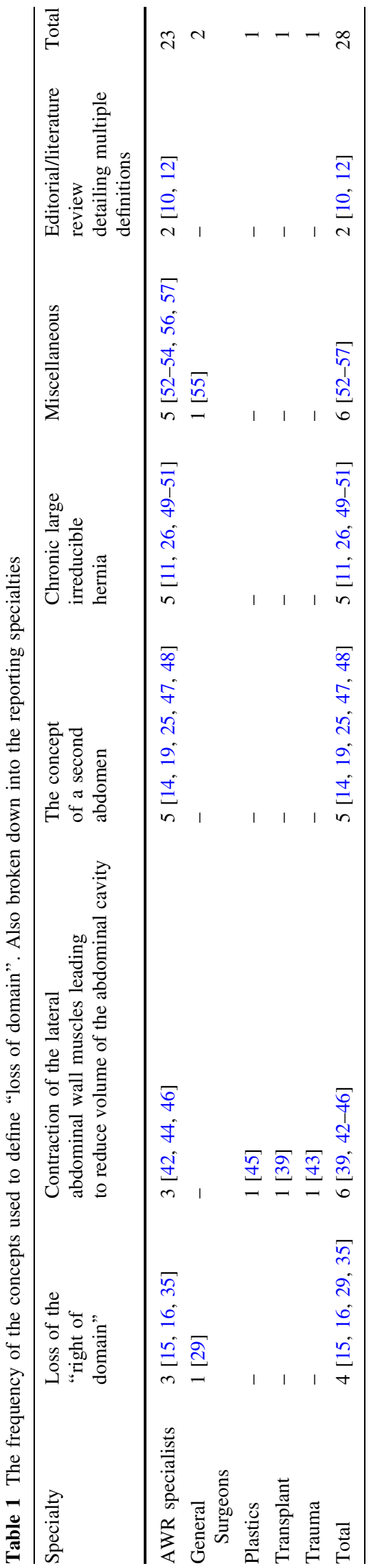

anatomical changes have physiological side effects. As intra-abdominal viscera herniate out of the abdominal cavity, intra-abdominal pressure reduces causing diaphragmatic descent and respiratory dysfunction. Portal venous stasis often occurs, causing mesenteric and bowel wall oedema, swelling the contents of the hernia sac making reduction even more challenging [26]. Venous stasis leads to congested bowel, ischaemic bowel, diarrhoea, and abdominal pain. Lastly, malalignment of the rectus muscles, atrophy of the strap muscles, and reduced intra-abdominal pressure results in an unsupported spine, precipitating chronic back pain. The pathological consequences of large ventral hernia were first described by Rives in 1973 and given the name "eventration disease" [62]. Given the clinical consequences of $\mathrm{CVH}$ and the difficulties of treatment, it is important that the metrics used to describe hernia morphology are relevant and consistent. Loss of domain would seem to be especially relevant, as it serves to describe the volumetric relationship between the hernia and the residual abdominopelvic cavity. However, our systematic review found that definitions are either not described or are disparate.

We found that current written definitions fell into six broad groupings. Two groups included six articles giving miscellaneous definitions [52-57] which could not categorised and two editorials listing multiple definitions $[10,12]$. The remaining four groups were based on four theoretical concepts. Some articles defined LOD as the loss of the "right of domain", a meaning that is unclear $[15,16,29,35]$. Interestingly, "right of domain" is a phrase used in UK common law and refers to a citizen's right to the ownership or possession of land. It is unclear how or when this phrase was used to refer to abdominal viscera; the earliest reference, we could find was from 1972. In this paper, Willard Johnson from Chelsea, Massachusetts, writes, "Infrequently a hernia is seen that has such a large sac that a significant portion of the abdominal viscera is residing outside the abdominal cavity. Over time no space is left in the abdomen to accommodate the replacement of such viscera. The contents of the sac have lost the 'right of domain' in the abdomen" [63]. Thus, this first definition suggests that the abdominal viscera lose the right to "belong" inside the abdominal cavity.

The second definition we identified is based on pathological processes that occur due to large abdominal defects [39, 42-46]. As described above, the abdominal strap muscles contract, shorten, and thicken. This definition uses the term "domain" to refer to abdominal cavity volume; contraction of the lateral strap muscles reduces the abdominal volume. Loss of domain, sometimes referred to as "loss of abdominal domain", in this case really means loss of abdominal cavity volume. 
Five articles used the term "loss of domain" without referring to abdominal cavity volume [14, 19, 25, 47, 48]. Perhaps the authors assume that readers are aware that "loss of domain" refers to "loss of abdominal volume"? Instead, these authors focus their definition on hernias being so large that a "second abdomen cavity" is created inside the hernia sac. Three out of these five articles [19, 25, 48] add an additional aspect to their definition mentioning the significant physiological difficulties that may occur if this "second abdomen" is reduced back into the patient's abdominal cavity. The origins of this description of the hernia sac as a "second abdomen" are unknown.

Lastly, five articles used definitions that appeared similar or equivalent to the definition of a large irreducible ventral hernia [11, 26, 49-51]. Previous manuscripts have noticed that definitions for LOD and irreducible hernia are sometimes not dissimilar [26]. These articles use terms like, "the volume of the hernia can no longer be reduced to the abdominal cavity" [26] and, "hernia contents are set by adhesions and not reducible to the abdominal cavity" [11]. Clearly a standardised definition should distinguish hernias with LOD alone from those with irreducible and incarcerated components. Finally, it is important to mention that 49 articles, $64 \%$ of the total, use the phrase "loss of domain" without any definition at all, or any reference to a standardised definition. Consequently, we must conclude that a knowledge or understanding of the concept of LOD is often assumed by authors despite there being no standardised definition.

We believe that a globally accepted written and volumetric definition for LOD is required so that it can be used as a predictor of operative outcomes. Necessarily, this will incorporate measurements of the hernia and residual abdominopelvic cavity. However, whilst such definitions exist already, these are not consistent and are based around two equations. In the first case, the hernia sac volume (HSV) is defined as a proportion of the abdominal cavity volume (ACV) (the Tanaka method, HSV/ACV [25]). This definition was used by eight studies in our systematic review $[11,25,26,44,46,51,58,59]$. The alternative is to describe hernia volume as a proportion of the total peritoneal volume (TPV) (the Sabbagh method, ACV + HSV = TPV, HSV/TPV [15]). This definition was used by five studies in our systematic review [14-16, 49, 50]. It is presently unclear which of these two definitions would be most appropriate or which operating surgeons feel would be the most meaningful and intelligible. The authors feel it is more logical and comprehensible to describe hernia volume as a proportion of total peritoneal volume, as this describes the percentage of abdominal viscera that has herniated. 
Furthermore, a future volumetric definition of LOD may include subtypes of hernia by incorporating hernia neck width into the classification. Large hernias with narrow necks present a different surgical challenge compared to those with wide necks. In clinical practice, abdominal wall surgeons use hernia morphology to decide upon surgical approach and reconstructive techniques. Similarly, the possible array of post-operative outcomes is likely to be dependent on hernia morphology. As yet a descriptor that distinguishes between subtypes of giant hernia by neck width, or any other parameter, does not exist and future work into this is warranted.

Establishing an internationally accepted classification for LOD is the next step. To facilitate this, we intend to carry out a Delphi consensus study working with academic hernia surgeons. Several of the surgeons on the Delphi panel will be leading members of the American Hernia Society, the British Hernia Society, the European Hernia Society, and the Asian and Pacific Hernia Society. During the rounds of voting, panellists will be presented with the four written definitions and two volumetric definitions discovered by this systematic review and asked to pick their preference. Panellists will be asked to suggest improvements and alterations to their chosen definition. We will challenge panellists to establish a standardised definition that can be applied to all hernia subtypes (i.e. giant inguinal, diaphragmatic, and ventral hernias). After publication of our classification, the authors will seek endorsement from the international hernia societies to aid propagation and acceptance of this classification.

\section{Conclusion}

Via systematic review, we have demonstrated that definitions of loss of domain are either disparate or omitted altogether. We found four broad concepts within the literature. Some were vague, and even the two volumetric definitions differed. Since loss of domain is a prime descriptor of hernia size and likely to be correlated with operative outcomes, a standardised definition is needed urgently.

Funding Funding has recently been received from the National Institute for Health Research and from Allergan PLC. Neither funders have been involved in the planning, methodology, analysis or write up of the research. National Institute of Health Research, Room 132, Richmond House, 79 Whitehall, London, SW1A 2NS: (Research for Patient Benefit programme, reference: PB-PG-0816-20005). Allergan Plc, Clonshaugh Business and Technology Park, Coolock, Dublin, D17 E400, Ireland.

\section{Compliance with ethical standards}

Conflict of interest Windsor A.C.J. declares conflicts of interest not directly related to the submitted work; educational Grants and speaker for: BARD, LifeCell and Cook. Parker S.G, Blackburn S, Plumb A.A, Archer L, Mallet S and Halligan S declare no conflicts of interest.

Open Access This article is distributed under the terms of the Creative Commons Attribution 4.0 International License (http://crea tivecommons.org/licenses/by/4.0/), which permits unrestricted use, distribution, and reproduction in any medium, provided you give appropriate credit to the original author(s) and the source, provide a link to the Creative Commons license, and indicate if changes were made.

\section{Appendix 1}

\section{Search strategy and string}

(((loss of domain) OR loss of abdominal domain)) AND hernia

Changed by the Pubmed search engine to

((loss[All Fields] AND ("domain"[All Fields])) AND

("hernia"[MeSH Terms] OR "hernia"[All Fields])

\section{Appendix 2}

For the purposes of this review, we defined publications as written by abdominal wall reconstruction surgeons if the following criteria were met: Inclusion criteria

1. The authors affiliation was to an "abdominal wall unit" or "hernia centre".

2. Manuscripts (not case reports) published using or describing complex abdominal wall reconstructive techniques (such as pre-operative pneumoperitoneum and pre-operative Botox therapy).

3. The centre had a well-known international reputation for AWR, known to the authors of this review.

4. AWR case series published by both general surgeons and plastic surgeons.

Exclusion criteria

1. Manuscripts published by centres clearly belonging to other specialities (e.g. Paediatrics, Trauma)

2. Case reports using complex reconstructive techniques that are written by authors not affiliated to a specialist "abdominal wall unit" or a "hernia centre" 


\section{References}

1. Shelton J, Poulose BK, Phillips S, Moore D, Nealon W, Penson D et al (2012) Epidemiology and cost of ventral hernia repair: making the case for hernia research. Hernia 16:179-183

2. Dabbas N, Adams K, Pearson K, Royle GT (2011) Frequency of abdominal wall hernias: is classical teaching out of date? J R Soc Med 2(1):1-6

3. Lutz W, Sanderson W, Scherbov S (2008) The coming acceleration of global population ageing. Nature 451:716-719

4. The Royal College of Physicians (2004) Storing up problems: the medical case for a slimmer nation

5. Hospital Episode Statistics (2017) UK ventral hernia data: report on request

6. Soop M, Carlson GL (2017) Recent developments in the surgical management of complex intra-abdominal infection. Br J Surg 104(2):e65-e74

7. Israelsson LA, Millbourn D (2013) Prevention of incisional hernias. How to close a midline incision. Surg Clin North Am 93(5):1027-1040

8. Vidović D, Jurišić D, Franjić BD, Glavan E, Ledinsky M, Bekavac-Bešlin M (2006) Factors affecting recurrence after incisional hernia repair. Hernia (Internet) 10:322-325. https://doi. org/10.1007/s10029-006-0097-z

9. Hauters P, Desmet J, Gherardi D, Dewaele S, Poilvache H, Malvaux P (2017) Assessment of predictive factors for recurrence in laparoscopic ventral hernia repair using a bridging technique. Surg Endosc (Internet). http://www.ncbi.nlm.nih.gov/pubmed/ 28078462\%0Ahttp://link.springer.com/10.1007/s00464-016-5401-0

10. Kirkpatrick AW, Nickerson D, Roberts DJ, Rosen MJ, McBeth $\mathrm{PB}$, Petro CC et al (2017) Intra-abdominal hypertension and abdominal compartment syndrome after abdominal wall reconstruction: quaternary syndromes? Scand J Surg (Internet). 106(2):97-106. https://doi.org/10.1177/1457496916660036

11. Passot G, Villeneuve L, Sabbagh C, Renard Y, Regimbeau J-M, Verhaeghe RK et al (2016) Definition of giant ventral hernias: development of standardization through a practice survey. Int $\mathbf{J}$ Surg 28:136-140

12. Halligan S, Parker SG, Plumb AA, Windsor ACJ (2018) Imaging complex ventral hernias, their surgical repair, and their complications. Eur Radiol 28:3560-3569

13. Liberati A, Altman DG, Tetzlaff J, Mulrow C, Gøtzsche PC, Ioannidis JPA et al (2009) The PRISMA statement for reporting systematic reviews and meta-analyses of studies that evaluate health care interventions: explanation and elaboration. J Clin Epidemiol 62(10):e1-e34

14. Renard Y, Lardiere-Deguette S, de Mestier L, Appere F, Colosio A, Kianmanesh R et al (2016) Management of large incisional hernias with loss of domain: a prospective series of patients prepared by progressive preoperative pneumoperitoneum. Surgery 160(2):426-435

15. Sabbagh C, Dumont F, Robert B, Badaoui R, Verhaeghe P, Regimbeau JM (2011) Peritoneal volume is predictive of tenisonfree closure of large incisional hernias with loss of domain: a prospective study. Hernia 15:559-565

16. Sabbagh C, Dumont F, Fuks D, Yzet T, Verhaeghe P, Regimbeau JM (2012) Progressive preoperative pneumoperitoneum preparation (the Goni Moreno protocol) prior to large incisional hernia surgery: volumetric, respiratory and clinical impacts. A prospective study. Hernia 16(1):33-40

17. Alyami M, Passot G, Voiglio E, Lundberg PW, Valette PJ, Muller A et al (2015) Feasibility of catheter placement under ultrasound guidance for progressive preoperative pneumoperitoneum for large incisional hernia with loss of domain. World $\mathbf{J}$
Surg 39(12):2878-2884. https://doi.org/10.1007/s00268-0153206-2

18. Skipworth JR, Vyas S, Uppal L, Floyd D, Shankar A (2014) Improved outcomes in the management of high-risk incisional hernias utilizing biological mesh and soft-tissue reconstruction: a single center experience. World J Surg 38(5):1026-1034. https:// doi.org/10.1007/s00268-013-2442-6

19. Kingsnorth AN, Sivarajasingham N, Wong S, Butler M (2004) Open mesh repair of incisional hernias with significant loss of domain. Ann R Coll Surg Engl 86(5):363-366

20. Parker SG, Wood CPJ, Butterworth JW, Boulton RW, Plumb AAO, Mallett $\mathrm{S}$ et al (2018) A systematic methodological review of reported perioperative variables, postoperative outcomes and hernia recurrence from randomised controlled trials of elective ventral hernia repair: clear definitions and standardised datasets are needed. Hernia (Internet). https://doi.org/10.1007/s10029017-1718-4

21. Cavalli M, Biondi A, Bruni PG, Campanelli G (2015) Giant inguinal hernia: the challenging hug technique. Hernia 19(5):775-783

22. Ferrari GC, Miranda A, Sansonna F, Magistro C, Di Lernia S, Maggoini D et al (2008) Laparoscopic management of incisional hernias $>$ or $=15 \mathrm{~cm}$ in diameter. Hernia 12(6):571-576

23. Punjani R, Shaikh I, Soni V (2015) Component separation technique: an effective way of treating large ventral hernia. Indian $\mathrm{J}$ Surg 77(Suppl 3):1476-1479

24. Dinesh HN, Kumar CJ, Shreyas N (2014) Giant inguinoscrotal hernia repaired by lichtensteins technique without loss of domain: a case report. J Clin Diagn Res 8(9):7-8

25. Tanaka EY, Yoo JH, Rodrigues AJJ, Utiyama EM, Birolini D, Rassian S (2010) A computerized tomography scan method for calculating the hernia sac and abdominal cavity volume in complex large incisional hernia with loss of domain. Hernia 14(1):63-69

26. Valezi AC, de Melo BGF, Marson AC, Liberatti M, Lopes AGJ (2018) Preoperative progressive pneumoperitoneum in obese patients with loss of domain hernias. Surg Obes Relat Dis 14(2):138-142

27. Serpell JW, Polglase AL, Anstee EJ (1988) Giant inguinal hernia. Aust N Z J Surg 58(10):831-834

28. King JN, Didlake RH, Gray RE (1986) Giant inguinal hernia. South Med J 79(2):252-253

29. Coopwood RW, Smith RJ (1989) Treatment of large ventral and scrotal hernias using preoperative pneumoperitoneum. J Natl Med Assoc 81(4):402-404

30. Schnitzer JJ, Kikiros CS, Short BL, O'Brien A, Anderson KD, Newman KD (1995) Experience with abdominal wall closure for patients with congenital diaphragmatic hernia repaired on ECMO. J Paediatr Surg 30(1):19-22

31. Harrison D, Taneja R, Kahn D, Rush BJ (1995) Repair of a massive ventral hernia in a morbidly obese patient. $\mathrm{N} J$ Med 92(6):387-389

32. Raynor RW, Del Guercio LR (1989) The place for pneumoperitoneum in the repair of massive hernia. World J Surg 13(5):581-585. https://doi.org/10.1007/BF01658874

33. Diamond S, Cryer HG (2015) Revising recommendations and outcome measurements after complex open abdominal wall reconstruction. Am Surg 81(10):955-960

34. Wink JD, Wes AM, Fischer JP, Nelson JA, Stranksy C, Kovach SJ 3rd (2015) Risk factors associated with early failure in complex abdominal wall reconstruction: a 5 year single surgeon experience. J Plast Surg Hand Surg 49(2):77-82

35. Hadad I, Small W, Dumanian GA (2009) Repair of massive ventral hernias with the separation of parts technique: reversal of the "lost domain". Am Surg 75(4):301-306 
36. Sandvall BK, Suver DW, Said HK, Mathes DW, Neligan PC, Dellinger EP et al (2016) Comparison of synthetic and biologic mesh in ventral hernia repair using components separation technique. Ann Plast Surg 76(6):674-679

37. Janis JE, O'Neill AC, Ahmad J, Zhong T, Hofer SO (2012) Acellular dermal matrices in abdominal wall reconstruction: a systematic review of the current evidence. Plast Reconstr Surg 130(5 Suppl 2):183s-193s

38. Gerlach UA, Pascher A (2012) Technical advances for abdominal wall closure after intestinal and multivisceral transplantation. Curr Opin Organ Transpl 17(3):258-267

39. Mangus RS, Kubal CA, Tector AJ, Fridell JA, Klingler K, Vianna RM (2012) Closure of the abdominal wall with acellular dermal allograft in intestinal transplantation. Am J Transpl 12(Suppl 4):s55-s59

40. Wartman SM, Woo K, Brewer M, Weaver FA (2017) Management of a large abdominal aortic aneurysm in conjunction with a massive inguinal hernia. Ann Vasc Surg 42:e302-e307

41. Borbely Y, Zerkowski J, Altemeier J, Eschenburg A, Kroll D, Nett P (2017) Complex hernias with loss of domain in morbidly obese patients: role of laparoscopic sleeve gastrectomy in a multistep approach. Surg Obes Relat Dis 13(5):768-773

42. Agnew SP, Small W, Wang E, Smith LJ, Hadad I, Dumanian GA (2010) Prospective measurements of intra-abdominal volume and pulmonary function after repair of massive ventral hernias with the components separation technique. Ann Surg (Internet) 251(5):981-988. http://meta.wkhealth.com/pt/pt-core/templatejournal/lwwgateway/media/landingpage.htm?issn=0003-4932\&vol ume $=251 \&$ issue $=5 \&$ spage $=981 \% 5$ Cnpapers $3: / /$ publication $/$ doi $/ 10$. 1097/SLA.0b013e3181d7707b

43. Dennis A, Vizinas TA, Joseph K, Kingsley S, Bokhari F, Starr F et al (2013) Not so fast to skin graft: transabdominal wall traction closes most "domain loss" abdomens in the acute setting. J Trauma Acute Care Surg 74(6):1486-1492

44. Bikchandani J, Fitzgibbons RJJ (2013) Repair of giant ventral hernias. Adv Surg 47:1-27

45. Tobias AM, Low DW (2003) The use of a subfascial vicryl mesh buttress to aid in the closure of massive ventral hernias following damage-control laparotomy. Plast Reconstr Surg 112(3):766-776

46. Oprea V, Matei O, Gheorghescu D, Leuca D, Buia F, Rosianu M et al (2014) Progressive preoperative pneumoperitoneum (PPP) as an adjunct for surgery of hernias with loss of domain. Chirugia (Bucur) 109(5):664-669

47. Van Geffen HJ, Simmermacher RK (2005) Incisional hernia repair: abdominoplasty, tissue expansion, and methods of augmentation. World J Surg 29(8):1080-1085. https://doi.org/10. 1007/s00268-005-7972-0

48. Berrevoet F, Martens T, Van Landuyt K, de Hemptinne B (2010) The anterolateral thigh flap for complicated abdominal wall reconstruction after giant incisional hernia repair. Acta Chir Belg 110(3):376-382

49. Mcadory RS, Cobb WS, Carbonell AM (2009) Progressive preoperative pneumoperitoneum for hernias with loss of domain. Am Surg 75(6):504-508
50. Azar FK, Crawford TC, Poruk KE, Farrow N, Cornell P, Nadra O et al (2017) Ventral hernia repair in patients with abdominal loss of domain: an observational study of one institution's experience. Hernia 21:245-252

51. Bueno-Lledo J, Torregrosa A, Jimenez R, Pastor PG (2018) Preoperative combination of progressive pneumoperitoneum and botulinum toxin type $\mathrm{A}$ in patients with loss of domain hernia. Surg Endosc 32:3599-3608

52. Zielinski MD, Goussous N, Schiller HJ, Jenkins D (2013) Chemical components separation with botulinum toxin A: a novel technique to improve primary fascial closure rates of the open abdomen. Hernia 17(1):101-107

53. Baghai M, Ramshaw BJ, Smith CD, Fearing N, Bachman S, Ramaswamy A (2009) Technique of laparoscopic ventral hernia repair can be modified to successfully repair large defects in patients with loss of domain. Surg Innov 16(1):38-45

54. King J, Hayes JD, Richmond B (2013) Repair of giant subcostal hernia using porcine acellular dermal matrix $\left(\right.$ Strattice $\left.^{\mathrm{TM}}\right)$ with bone anchors and pedicled omental flap coverage: a case report. J Med Case Rep 11(7):258

55. Hamad A, Marimuthu K, Mothe B, Hanafy M (2013) Repair of massive inguinal hernia with loss of abdominal domain using laparoscopic component separation technique. J Surg Case Rep 2013(3):1-3

56. Fernando EJ, Guerron AD, Rosen MJ (2015) A case of splenic rupture within an umbilical hernia with loss of domain. Gastrointest Surg. 19(4):789-791

57. Mayagoitia JC, Suarez D, Arenas JC, Daiz de Leon V (2006) Preoperative progressive pneumoperitoneum in patients with abdominal-wall hernias. Hernia 10(3):213-217

58. Petro CC, Raigani S, Fayezizadeh M, Rowbottom JR, Klick JC, Prabhu AS et al (2015) Permissible intraabdominal hypertension following complex abdominal wall reconstruction. Plast Reconstr Surg 136(4):868-881

59. Afifi RY, Hamood M, Hassan M (2018) The outcome of A. Double mesh intraperitoneal repair for complex ventral hernia: a retrospective cohort study. Int J Surg 53:129-136

60. Elstner KE, Read JW, Rodriguez-Acevedo O, Ho-Shon K, Magnussen J, Ibrahim N (2017) Preoperative progressive pneumoperitoneum complementing chemical component relaxation in complex ventral hernia repair. Surg Endosc 31(4):1914-1922

61. Martin AE, Khan A, Kim DS, Muratore CS, Luks FI (2009) The use of intraabdominal tissue expanders as a primary strategy for closure of giant omphaloceles. J Paediatr Surg 44(1):178-182

62. Rives J, Lardennois B, Pire JC, Hibon J (1973) Les grandes eventrations. Importance du "volet abdominal" et des troubles respiratiores qui luis sont secondaires. Chirurgia (Bucur) 99:547-563

63. Johnson WC (1972) Preoperative progressive pneumoperitoneum in preparation for repair of large hernias of the abdominal wall. Am J Surg 124:63-68 